\title{
A Deep Learning Framework for Coronavirus Disease (COVID-19) Detection in X-Ray Images
}

Tayyip Ozcan ( $\nabla$ tozcan@erciyes.edu.tr)

Erciyes University https://orcid.org/0000-0002-3111-5260

\section{Research Article}

Keywords: COVID-19 detection, X-Ray images, Deep learning, CNN, Transfer learning, Hyperparameter optimization, Grid search

Posted Date: May 5th, 2020

DOl: https://doi.org/10.21203/rs.3.rs-26500/v1

License: (9) This work is licensed under a Creative Commons Attribution 4.0 International License. Read Full License 


\title{
A Deep Learning Framework for Coronavirus Disease (COVID-19) Detection in X-Ray Images
}

\author{
Tayyip Ozcan
}

Received: date / Accepted: date

\begin{abstract}
Coronavirus, a large family of viruses, causes illness in both humans and animals. The novel coronavirus (COVID-19) came up in Wuhan in December 2019. This deadly COVID-19 pandemic has become very fast-spreading and currently present in several countries worldwide. The timely detection of patients who have COVID-19 is vitally important. To this end, scientists are working on different detection methods.In this paper, a grid search (GS) and pre-trained model aided convolutional neural network (CNN) model is proposed to detect COVID-19 in X-Ray images. In the proposed method, the GS method is employed to optimize the hyperparameters of CNN, which directly affects classification performance. Three pre-trained CNN models (GoogleNet, ResNet18 and ResNet50), which can be used for classification, feature extraction and transfer learning purposes were used for transfer learning in this study. The proposed method was trained using the training and validation subdatasets of the collected dataset and detail evaluations are presented according to different performance metrics. According to the experimental studies, the best results were obtained with the GS and ResNet50 aided model.
\end{abstract}

Keywords COVID-19 detection · X-Ray images · Deep learning · CNN . Transfer learning . Hyperparameter optimization · Grid search

\section{T. Ozcan}

E-mail: tozcan@erciyes.edu.tr

Dept. of Computer Engineering, Erciyes University, 38039,

Melikgazi, Kayseri, Turkey

Phone: +90 (352) 207-6666

Fax: +90 (352) 437-5784

\section{Introduction}

COVID-19 is a fast-spreading lethal coronavirus. It has spread all over the world in a very short time since it was initially detected in December 2019. Symptoms of this deadly include fever, dry cough and weakness [15]. The rapid and timely detection of sick individuals is important for both the patient's health and the prevention the further spread of the virus. COVID-19 diagnoses can be made by swabs from the nose and throat, blood count, computed tomography and X-Ray images.

Convolutional neural networks (CNNs), which have basic functions such as object recognition, classification and regression, has been popular in recent years. Some researchers have employed CNN for the detection of COVID-19. Wang and Wong [36] developed a COVID-NET with a deep CNN architecture for COVID-19 detection. They also presented the COVIDx dataset, which is adopted in this study. According to their experimental results, their proposed method produced good results.

A transfer learning-based CNN model that classifies medical data into three categories (normal, pneumonia, and Covid-19) has been proposed by Apostolopoulos and Bessiana [3]. In their study, which was carried out using five different pre-trained CNN models, they reported an accuracy of $98.75 \%$ for the binarry classification problem and $93.48 \%$ for the multiclassification problem. Hemdan et al. [10] investigated COVIDX-19 classification with 7 different deep CNN architectures. In their experimental studies, they worked on two different categories, COVID-19 and non-COVID-19. Their dataset consisted of a total of 50 images. They reportedly achieved better results with the VGG19 and DenseNet201 models. Ghoshal 
and Tucker [11] proposed a Dropweights based Bayesian Convolutional Neural Networks model and tested the proposed model for 4 different classes (normal, bacterial pneumonia, non-COVID-19 viral pneumonia and COVID-19). Sethy and Behera [32] extracted features from X-Ray images using pre-trained moldels. They collected their dataset from GitHub, Kaggle, and Open-I repository. According to their experimental results, features obtained from ResNet50 model and subsequently classified with SVM produced more successful results than the other models. A new deep learning aided anomaly detection model for COVID-19 detection from X-Ray images was proposed by Zhang et al. [39]. Their model achieved a sensitivity of $90.00 \%$ specificity of $87.84 \%$ when the threshold was set to 0.25. Narin et al. [21] proposed a transfer learning-based $\mathrm{CNN}$ model for detection of COVID-19. They used ResNet50, InceptionV3, and Inception- ResNetV2 pre-trained models for transfer learning. According to the results of their simulations, the ResNet50 based model recorded the best results.

As seen in the above literature review for COVID-19, the use of transfer learning based CNN is important to produce an effective model. Transfer learning supported CNN model has also used for different problems $[24,22,26,23,6]$. Similarly, pre-trained models have been used in the literature for different purposes besides transfer learning $[1,2,4,12,35,37]$.

The optimization of hyperparameters is an effective way to obtain higher success rates for machine learning methods. For this purpose, there is a plethora of studies in the literature. In addition to heuristic optimization algorithms [30, 17, 38, 8, 14, 25, 26, 24], the global search methods $[31,14,29,7]$ and other methods $[9,28]$ have also been used for hyperparameter optimization.

This study carries out the classification of COVID-19 from X-Ray images by using transfer learning-based CNN, whose hyperparameters were optimized by the GS method.

In the first stage, a dataset was created using $\mathrm{X}$-Ray images collected from different platforms. This dataset includes bacteria, COVID-19, normal and virus classes. In the next stage, the proposed classification method was evaluated with the dataset and the detailed results are presented according to different performance metrics.

The main contributions of the paper are given below:

- In this paper, a transfer learning based CNN is proposed for COVID-19 detection on X-Ray images. The hyperparameters of the generated CNN models are tuned by using the GS method. To the best of our knowledge, this study is the first in the literature that utilizes this approach for COVID-19 detection.

- The GS and ResNet18 aided method have the smallest computational time for parameter optimization.

- The GS and ResNet50 aided method obtained the best performance based on all of the metrics (accuracy, sensvitiy, specifity, precision, and F1_score).

The remainder of this paper is organized as follows. The methodology including CNN, pre-trained models, GS, and the proposed method is given in Section 2. The experimental setup and results are provided in Section 3. Finally, Section 4 presents the conclusion and future works.

\section{Methodology}

In this paper, a deep learning model based unified method is presented for COVID-19 detection in X-Ray images. In the method section, convolutional neural networks (CNN) [18], which is a deep learning method, is used as the classifier and GoogleNet [34], ResNet18 [13], ResNet50 [13] pre-trained models are used for transfer learning. Additionally, a grid search method, which is a global search algorithm, is used for optimization of the the training settings of the generated CNN model. Al detailed description is presented below.

\subsection{Convolutional Neural Networks}

CNN, which is a multi-layer neural network [19], is a type of artificial neural network and it is specialized to process big data [18]. A basic CNN structure usually has convolution, pooling, flattening, and fully connected layers. An example of a CNN architecture is presented in Fig. 1.

The convolution layer contains a set of learnable filters and is one of the structures in which the learning skill of CNN takes place [26]. An example of convolution operation is given in Fig. 2. Here, $\mathbf{I}$ refers to input image, $\mathbf{K}$ refers to filter and $\mathbf{I}^{*} \mathbf{K}$ refers to feature map.

Another important structure that reduces the computational cost of a network is the pooling layer. Maximum, average and minimum pooling are the most frequently used pooling techniques [16]. An example of maxpooling operation is given in Fig. 3. 


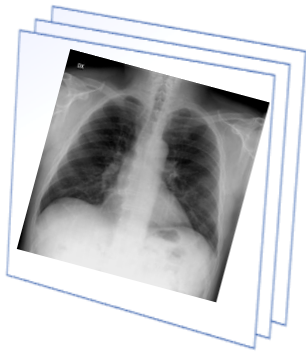

Input

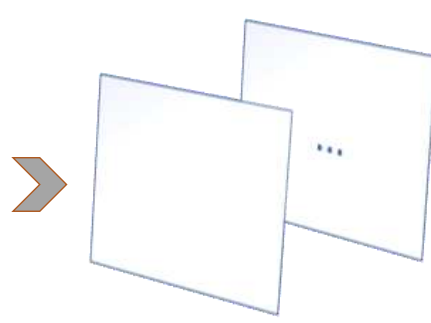

Convolution

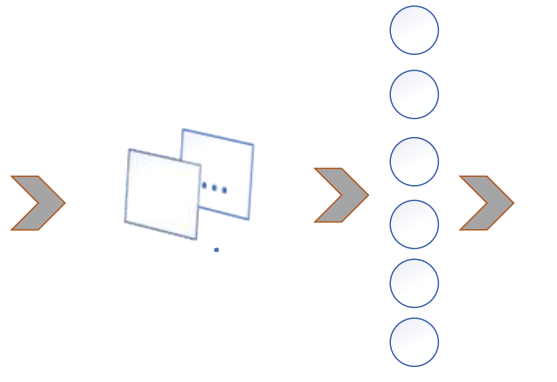

Pooling

\section{Bacteria \\ Covid \\ Normal \\ Virus}

Output

Fig. 1: The main layers of a CNN architecture

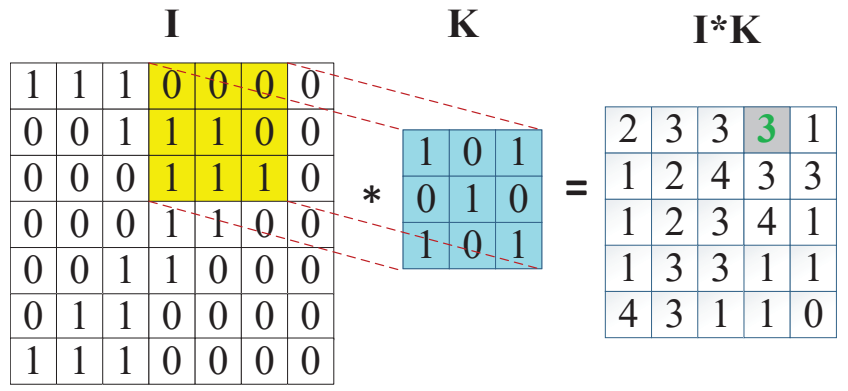

Fig. 2: An example of the convolution operation.

\begin{tabular}{|c|c|c|c|c|c|c|}
\hline 11 & 12 & 11 & 15 & & & \\
\hline 7 & 4 & 12 & 9 & $2 \times 2$ Max-Pool & 12 & 15 \\
\hline 3 & 15 & 25 & 15 & & 18 & 25 \\
\hline 18 & 13 & 4 & 12 & & & \\
\hline
\end{tabular}

Fig. 3: An example of the maxpooling operation.

The fully connected layer comes after the both convolution and pooling layers of a CNN model [22]. Neurons in this layer have a full connection with the neurons in a previous layer. The data is flattened before coming to the fully connected layer. An example of a flattening operation is given in Fig. 4 .

The training options of the CNN have an effect on its successful performance. Minibatch size, initial learning rate, $\ell_{2}$ regularization parameter, and momentum value, which are optimized by GS in this paper, are some of the most important hyperparameters of a CNN model. The description of these hyperparameters are shown in Table 1.

Developing a new CNN model is a very challenging and time-consuming process. Using pre-trained models is a faster and more effective approach than

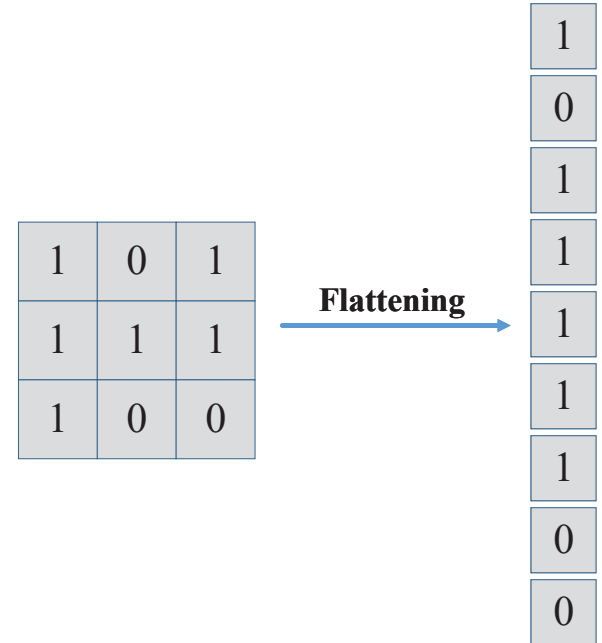

Fig. 4: An example of the flattening operation.

developing a new CNN model [23]. Pre-trained models are typically used for classification, transfer learning, and feature extraction. GoogleNet, ResNet18, and ResNet50 models were selected for transfer learning in this study. Some features of related pre-trained models are as shown in Table 2 .

Table 2: Some features of the pre-trained models [20]

\begin{tabular}{ccccc}
\hline Model & Depth & Size & $\begin{array}{c}\text { Parameters } \\
\text { (Millions) }\end{array}$ & $\begin{array}{c}\text { Image } \\
\text { Input Size }\end{array}$ \\
\hline GoogleNet & 22 & $27 \mathrm{MB}$ & 7.0 & 224 -by-224 \\
ResNet18 & 18 & $44 \mathrm{MB}$ & 11.7 & 224 -by-224 \\
ResNet50 & 50 & $96 \mathrm{MB}$ & 25.6 & 224 -by-224 \\
\hline
\end{tabular}

\subsection{Grid Search Algorithm}

The grid search (GS) method used to optimize the parameters of a model is frequently preferred in applications where a short process of optimization is 
Table 1: A brief description of the training options of a CNN model [24]

\begin{tabular}{|c|c|c|c|c|}
\hline Option & Description & Value & Default & Type \\
\hline Minibatch Size & Mini-batch size used for each training iteration. & Powers of 2 (Suggested) & 128 & Integer \\
\hline Initial Learning Rate & $\begin{array}{l}\text { Initial value of learning rate. If it is selected too low, } \\
\text { training will take a long time, but if it is selected too high, the } \\
\text { optimal result may not be found. }\end{array}$ & $(0,1)$ & 0.01 & Floating point \\
\hline$\ell_{2}$ regularization & $\begin{array}{l}\text { Value of } \ell_{2} \text { regularizer. Each set of parameters } \\
\text { in a layer can specify a multiplier for the } \ell_{2} \text { regularizer. }\end{array}$ & $(0,1)$ & 0.0001 & Floating point \\
\hline Momentum & $\begin{array}{l}\text { The contribution of the gradient step from the previous iteration } \\
\text { to the current iteration }\end{array}$ & $(0,1)$ & 0.90 & Floating point \\
\hline
\end{tabular}

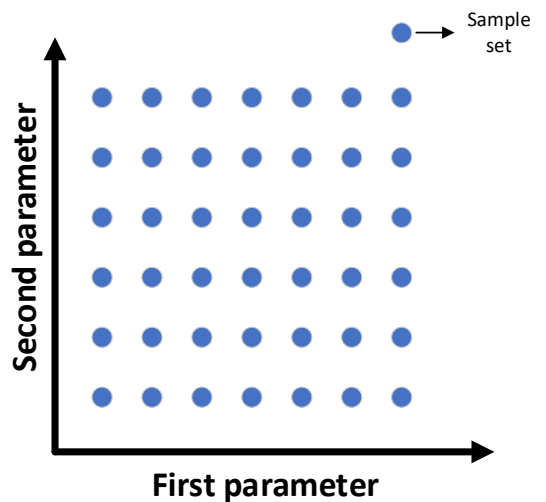

Fig. 5: Parameter sets in the search space for GS

desired. In GS, possible different values are determined in the related value ranges for a parameters. All combinations of these values (as shown in Fig. 5) are fed to the relevant model and the optimization process is started. The parameter set that gives the best results is determined and given as the output of the GS method.

\subsection{Proposed Method}

In this paper, a method including GS and transfer learning supported CNN model is proposed. The proposed method is given in Fig. 6. For the first step, the collected dataset is randomly split into three sub-datasets, which are training, validation, and testing. The training, validation, and testing sub-datasets are selected as $50 \%, 20 \%$, and $30 \%$ of the dataset, respectively. For the next phase, parameter optimization using GS is realized. In this phase, the optimally selected hyperparameters from the parameter set table, and training data are given to the transfer learning supported CNN model as input. Afterwards, the model is trained using the training data and these parameters. A trained network is formed as an output. The trained network is evaluated on validation data using the Eq. 1. The computed fitness function value (fval), and the hyperparameters are stored into a table ???. After the last loop of the parameter is realized, the best hyperparameters, which give the minimum fval value, are given as output of the parameter optimization phase. The best parameter set, training, and validation data are fed to the transfer learning CNN model. The final trained network serves as the output. Lastly, the trained network is evaluated on testing data and the result including all performance metrics is presented.

fval $=1-\frac{\text { successful prediction count }}{\text { total sample count }}$

\section{Experimental Studies}

In this section, the proposed method is applied on the collected dataset. The experimental setup is presented in Subsection 3.1 whereas the results of the proposed method are presented in Subsection 3.2.

\subsection{Experimental Setup}

The experimental setup phase was carried out before the main simulation stage. The data collection, parameter setup, environmental setup, and performance metrics are sub parts of this subsection.

\subsubsection{Data Collection}

Since the COVID-19 appeared in December 2019, it is quite difficult to obtain a public dataset for research work. Therefore, the data was obtained from different sources [5, 27] and hospital [33] shares. The number of samples for each class is given in Table 3 .

\subsubsection{Parameter Setup}

The initial learning rate (ILR), $\ell_{2}$ regularization $\left(\ell_{2} \mathrm{R}\right)$, momentum (Mom), and minibatch size (MiB) parameter values to be used in grid search are shown in Table 4. 


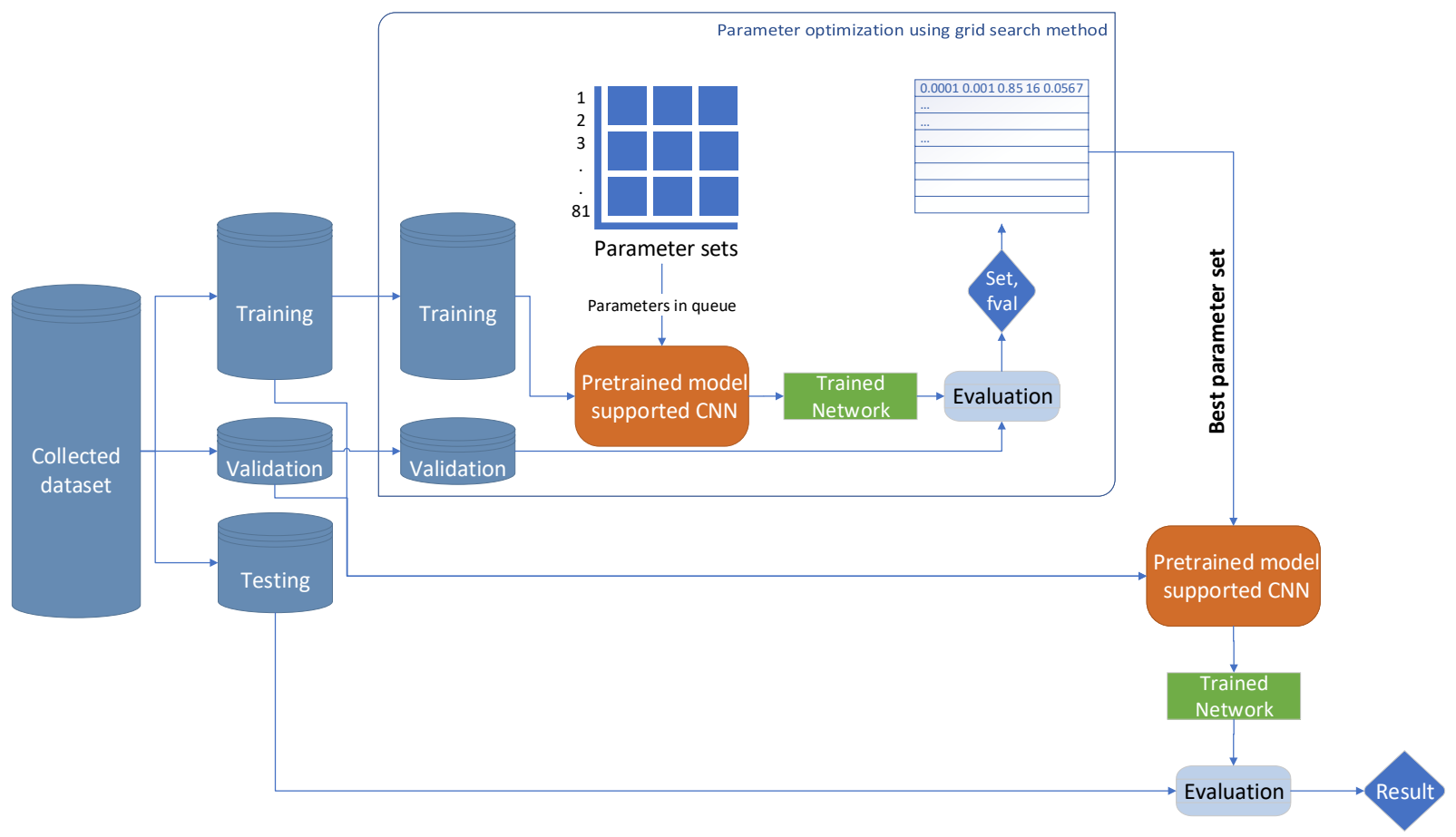

Fig. 6: The GS and Pre-trained model aided method

Table 3: Overview of the number of samples for each category in the dataset.

\begin{tabular}{lcccc}
\hline & Bacteria & Covid-19 & Normal & Virus \\
\hline Train & 121 & 66 & 100 & 74 \\
Validation & 48 & 26 & 40 & 30 \\
Test & 73 & 39 & 60 & 44 \\
\hline Total & 242 & 131 & 200 & 148 \\
\hline
\end{tabular}

Table 4: Parameter values used for GS

\begin{tabular}{cccc}
\hline ILR & $\ell_{2} \mathbf{R}$ & Mom & MiB \\
\hline 0.001 & 0.0001 & 0.85 & 8 \\
0.005 & 0.001 & 0.90 & 16 \\
0.010 & 0.005 & 0.95 & 32 \\
\hline
\end{tabular}

\subsubsection{Environmental Setup}

An Nvidia GTX 1080 Ti Turbo with 11 GB Ram capacity was used for the experimental simulations.

\subsubsection{Performance Metrics}

Different types of metrics were used in order to evaluate performance for the proposed models. True positives (TP), true negatives (TN), false positives $(\mathrm{FP})$, and false negatives $(\mathrm{FN})$ are used to calculate the metrics. Here, while TP refers to the correct predictions of COVID-19, TN refers to bacteria, normal or virus cases that were classified as non-COVID-19. Also, FP refers to bacteria, normal or virus cases that were classified as COVID-19. Lastly, FN refers to COVID-19 cases that classified as bacteria, normal or virus cases.

The performance metrics, which are accuracy, sensitivity (recall), specificity, precision, and F1-score, are calculated using Eq. 2-6, respectively.

Accuracy $=\frac{T P+T N}{T P+F P+T N+F N}$

Sensitivity $=\frac{T P}{T P+F N}$

Specificity $=\frac{T N}{T N+F P}$

Precision $=\frac{T P}{T P+F P}$

$F 1 \_$score $=2 \times \frac{\text { Precision } \times \text { Sensitivity }}{\text { Precision }+ \text { Sensitivity }}$ 


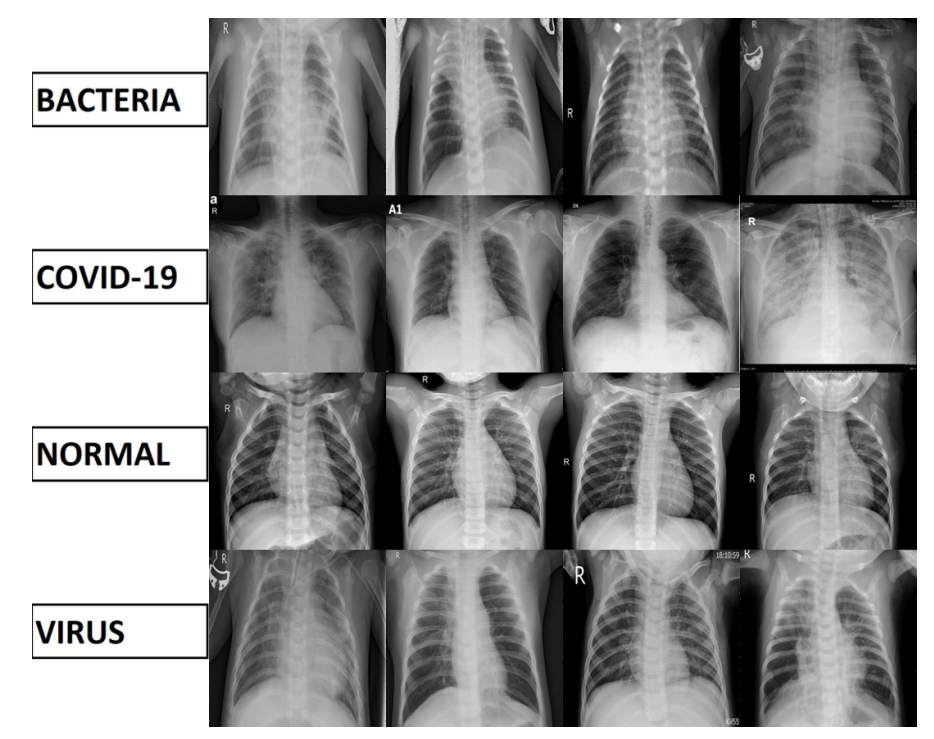

Fig. 7: Sample images from collected dataset

\subsection{Experimental Results}

\subsubsection{Parameter Optimization using GS for the Pre-trained Aided Model}

In the proposed method, the GS algorithm is used to optimize the training parameters of the CNN model. When Table 8 is examined, all combinations of parameter sets and validation errors (fitness value, (fval)) of these parameter sets are presented for GoogleNet aided proposed method. The parameter values giving the error of 0,0278 are the best hyperparameters kept in the memory as a result of the GS approach.

All combinations of parameter sets and fvals of these parameter sets are presented in Table 9 for ResNet18 aided proposed method. The parameter values giving the error of 0,0208 are the best hyperparameters kept in the memory.

For the last method, ResNet50 aided proposed method, all combinations of parameter sets and fval values of these parameter sets are presented in Table 10. The parameter values giving the error of 0,0278 are the best hyperparameters.

\subsubsection{General Results}

The cost of computational time for parameter optimization using GS is given in Table 5. According to the table, less time is required for parameter optimization of the ResNet18 aided method.
Table 5: Time cost (sec.) for parameter optimization

\begin{tabular}{ccc}
\hline GoogleNet & ResNet18 & ResNet50 \\
\hline 9675.1 & 8395.5 & 13374.9 \\
\hline
\end{tabular}

While the optimal hyperparameters obtained by GS are presented in Table 6, the performance comparison of the proposed methods on the whole system is given in Table 7 . When the results in the table is analyzed, the ResNet50 aided proposed method is the most successful one according to the metrics.

Table 6: The optimal hyperparameters selected by GS

\begin{tabular}{cccc}
\hline Parameters & GoogleNet & ResNet18 & ResNet50 \\
\hline ILR & 0.0050 & 0.0100 & 0.0050 \\
$\ell_{2} \mathrm{R}$ & 0.0010 & 0.0050 & 0.0001 \\
$\mathrm{Mom}$ & 0.90 & 0.85 & 0.85 \\
$\mathrm{MiB}$ & 32 & 16 & 16 \\
\hline
\end{tabular}

Table 7: Performance comparison of methods on whole system

\begin{tabular}{lccc}
\hline Metric (\%) & GoogleNet & ResNet18 & ResNet50 \\
\hline Accuracy & 93.98 & 97.22 & $\mathbf{9 7 . 6 9}$ \\
Sensitivity & 91.78 & $\mathbf{9 7 . 2 6}$ & $\mathbf{9 7 . 2 6}$ \\
Specificity & 95.10 & 97.20 & $\mathbf{9 7 . 9 0}$ \\
Precision & 90.54 & 94.67 & $\mathbf{9 5 . 9 5}$ \\
F1_score & 91.16 & 95.95 & $\mathbf{9 6 . 6 0}$ \\
\hline
\end{tabular}


Table 8: Hyperparameters and fval values for the GS and GoogleNet aided method

Table 9: Hyperparameters and fval values for the GS and ResNet18 aided method

\begin{tabular}{|c|c|c|c|c|c|}
\hline Run & ILR & $\ell_{2} \mathbf{R}$ & Mom & MiB & fval \\
\hline 1 & 0.0010 & 0.0001 & 0.85 & 8 & 0.1319 \\
\hline 2 & 0.0010 & 0.0001 & 0.85 & 16 & 0.0694 \\
\hline 3 & 0.0010 & 0.0001 & 0.85 & 32 & 0.0556 \\
\hline 4 & 0.0010 & 0.0001 & 0.90 & 8 & 0.0347 \\
\hline 5 & 0.0010 & 0.0001 & 0.90 & 16 & 0.0833 \\
\hline 6 & 0.0010 & 0.0001 & 0.90 & 32 & 0.0764 \\
\hline 7 & 0.0010 & 0.0001 & 0.95 & 8 & 0.0486 \\
\hline 8 & 0.0010 & 0.0001 & 0.95 & 16 & 0.0417 \\
\hline 9 & 0.0010 & 0.0001 & 0.95 & 32 & 0.0417 \\
\hline 10 & 0.0010 & 0.0050 & 0.85 & 8 & 0.0556 \\
\hline 11 & 0.0010 & 0.0050 & 0.85 & 16 & 0.1042 \\
\hline 12 & 0.0010 & 0.0050 & 0.85 & 32 & 0.1042 \\
\hline 13 & 0.0010 & 0.0050 & 0.90 & 8 & 0.0694 \\
\hline 14 & 0.0010 & 0.0050 & 0.90 & 16 & 0.0347 \\
\hline 15 & 0.0010 & 0.0050 & 0.90 & 32 & 0.0486 \\
\hline 16 & 0.0010 & 0.0050 & 0.95 & 8 & 0.0486 \\
\hline 17 & 0.0010 & 0.0050 & 0.95 & 16 & 0.0903 \\
\hline 18 & 0.0010 & 0.0050 & 0.95 & 32 & 0.0694 \\
\hline 19 & 0.0010 & 0.0010 & 0.85 & 8 & 0.1181 \\
\hline 20 & 0.0010 & 0.0010 & 0.85 & 16 & 0.0347 \\
\hline 21 & 0.0010 & 0.0010 & 0.85 & 32 & 0.0764 \\
\hline 22 & 0.0010 & 0.0010 & 0.90 & 8 & 0.0625 \\
\hline 23 & 0.0010 & 0.0010 & 0.90 & 16 & 0.1944 \\
\hline 24 & 0.0010 & 0.0010 & 0.90 & 32 & 0.0694 \\
\hline 25 & 0.0010 & 0.0010 & 0.95 & 8 & 0.1111 \\
\hline 26 & 0.0010 & 0.0010 & 0.95 & 16 & 0.1319 \\
\hline 27 & 0.0010 & 0.0010 & 0.95 & 32 & 0.0625 \\
\hline 28 & 0.0050 & 0.0001 & 0.85 & 8 & 0.2639 \\
\hline 29 & 0.0050 & 0.0001 & 0.85 & 16 & 0.1319 \\
\hline 30 & 0.0050 & 0.0001 & 0.85 & 32 & 0.0833 \\
\hline 31 & 0.0050 & 0.0001 & 0.90 & 8 & 0.3403 \\
\hline 32 & 0.0050 & 0.0001 & 0.90 & 16 & 0.0764 \\
\hline 33 & 0.0050 & 0.0001 & 0.90 & 32 & 0.1389 \\
\hline 34 & 0.0050 & 0.0001 & 0.95 & 8 & 0.6667 \\
\hline 35 & 0.0050 & 0.0001 & 0.95 & 16 & 0.6667 \\
\hline 36 & 0.0050 & 0.0001 & 0.95 & 32 & 0.1389 \\
\hline 37 & 0.0050 & 0.0050 & 0.85 & 8 & 0.0764 \\
\hline 38 & 0.0050 & 0.0050 & 0.85 & 16 & 0.0486 \\
\hline 39 & 0.0050 & 0.0050 & 0.85 & 32 & 0.0903 \\
\hline 40 & 0.0050 & 0.0050 & 0.90 & 8 & 0.1250 \\
\hline 41 & 0.0050 & 0.0050 & 0.90 & 16 & 0.6667 \\
\hline 42 & 0.0050 & 0.0050 & 0.90 & 32 & 0.1806 \\
\hline 43 & 0.0050 & 0.0050 & 0.95 & 8 & 0.6667 \\
\hline 44 & 0.0050 & 0.0050 & 0.95 & 16 & 0.5625 \\
\hline 45 & 0.0050 & 0.0050 & 0.95 & 32 & 0.0556 \\
\hline 46 & 0.0050 & 0.0010 & 0.85 & 8 & 0.1736 \\
\hline 47 & 0.0050 & 0.0010 & 0.85 & 16 & 0.1528 \\
\hline 48 & 0.0050 & 0.0010 & 0.85 & 32 & 0.1319 \\
\hline 49 & 0.0050 & 0.0010 & 0.90 & 8 & 0.6181 \\
\hline 50 & 0.0050 & 0.0010 & 0.90 & 16 & 0.0347 \\
\hline 51 & 0.0050 & 0.0010 & 0.90 & 32 & 0.0278 \\
\hline 52 & 0.0050 & 0.0010 & 0.95 & 8 & 0.6667 \\
\hline 53 & 0.0050 & 0.0010 & 0.95 & 16 & 0.6667 \\
\hline 54 & 0.0050 & 0.0010 & 0.95 & 32 & 0.1736 \\
\hline 55 & 0.0100 & 0.0001 & 0.85 & 8 & 1.0000 \\
\hline 56 & 0.0100 & 0.0001 & 0.85 & 16 & 0.5625 \\
\hline 57 & 0.0100 & 0.0001 & 0.85 & 32 & 0.5972 \\
\hline 58 & 0.0100 & 0.0001 & 0.90 & 8 & 1.0000 \\
\hline 59 & 0.0100 & 0.0001 & 0.90 & 16 & 0.6667 \\
\hline 60 & 0.0100 & 0.0001 & 0.90 & 32 & 0.5833 \\
\hline 61 & 0.0100 & 0.0001 & 0.95 & 8 & 1.0000 \\
\hline 62 & 0.0100 & 0.0001 & 0.95 & 16 & 0.6667 \\
\hline 63 & 0.0100 & 0.0001 & 0.95 & 32 & 0.6667 \\
\hline 64 & 0.0100 & 0.0050 & 0.85 & 8 & 0.6667 \\
\hline 65 & 0.0100 & 0.0050 & 0.85 & 16 & 0.2778 \\
\hline 66 & 0.0100 & 0.0050 & 0.85 & 32 & 0.1944 \\
\hline 67 & 0.0100 & 0.0050 & 0.90 & 8 & 1.0000 \\
\hline 68 & 0.0100 & 0.0050 & 0.90 & 16 & 0.6667 \\
\hline 69 & 0.0100 & 0.0050 & 0.90 & 32 & 0.6181 \\
\hline 70 & 0.0100 & 0.0050 & 0.95 & 8 & 1.0000 \\
\hline 71 & 0.0100 & 0.0050 & 0.95 & 16 & 1.0000 \\
\hline 72 & 0.0100 & 0.0050 & 0.95 & 32 & 0.6667 \\
\hline 73 & 0.0100 & 0.0010 & 0.85 & 8 & 0.6667 \\
\hline 74 & 0.0100 & 0.0010 & 0.85 & 16 & 0.4792 \\
\hline 75 & 0.0100 & 0.0010 & 0.85 & 32 & 0.6667 \\
\hline 76 & 0.0100 & 0.0010 & 0.90 & 8 & 1.0000 \\
\hline 77 & 0.0100 & 0.0010 & 0.90 & 16 & 1.0000 \\
\hline 78 & 0.0100 & 0.0010 & 0.90 & 32 & 1.0000 \\
\hline 79 & 0.0100 & 0.0010 & 0.95 & 8 & 1.0000 \\
\hline 80 & 0.0100 & 0.0010 & 0.95 & 16 & 0.6667 \\
\hline 81 & 0.0100 & 0.0010 & 0.95 & 32 & 0.6667 \\
\hline
\end{tabular}

\begin{tabular}{|c|c|c|c|c|c|}
\hline Run & ILR & $\ell_{2} \mathbf{R}$ & Mom & MiB & fval \\
\hline 1 & 0.0010 & 0.0001 & 0.85 & 8 & 0.0556 \\
\hline 2 & 0.0010 & 0.0001 & 0.85 & 16 & 0.0556 \\
\hline 3 & 0.0010 & 0.0001 & 0.85 & 32 & 0.0556 \\
\hline 4 & 0.0010 & 0.0001 & 0.90 & 8 & 0.0556 \\
\hline 5 & 0.0010 & 0.0001 & 0.90 & 16 & 0.0694 \\
\hline 6 & 0.0010 & 0.0001 & 0.90 & 32 & 0.0764 \\
\hline 7 & 0.0010 & 0.0001 & 0.95 & 8 & 0.0347 \\
\hline 8 & 0.0010 & 0.0001 & 0.95 & 16 & 0.0694 \\
\hline 9 & 0.0010 & 0.0001 & 0.95 & 32 & 0.0486 \\
\hline 10 & 0.0010 & 0.0050 & 0.85 & 8 & 0.0694 \\
\hline 11 & 0.0010 & 0.0050 & 0.85 & 16 & 0.1042 \\
\hline 12 & 0.0010 & 0.0050 & 0.85 & 32 & 0.1042 \\
\hline 13 & 0.0010 & 0.0050 & 0.90 & 8 & 0.0764 \\
\hline 14 & 0.0010 & 0.0050 & 0.90 & 16 & 0.0625 \\
\hline 15 & 0.0010 & 0.0050 & 0.90 & 32 & 0.0764 \\
\hline 16 & 0.0010 & 0.0050 & 0.95 & 8 & 0.0486 \\
\hline 17 & 0.0010 & 0.0050 & 0.95 & 16 & 0.0556 \\
\hline 18 & 0.0010 & 0.0050 & 0.95 & 32 & 0.0556 \\
\hline 19 & 0.0010 & 0.0010 & 0.85 & 8 & 0.0833 \\
\hline 20 & 0.0010 & 0.0010 & 0.85 & 16 & 0.0694 \\
\hline 21 & 0.0010 & 0.0010 & 0.85 & 32 & 0.8194 \\
\hline 22 & 0.0010 & 0.0010 & 0.90 & 8 & 0.0694 \\
\hline 23 & 0.0010 & 0.0010 & 0.90 & 16 & 0.0764 \\
\hline 24 & 0.0010 & 0.0010 & 0.90 & 32 & 0.0694 \\
\hline 25 & 0.0010 & 0.0010 & 0.95 & 8 & 0.0556 \\
\hline 26 & 0.0010 & 0.0010 & 0.95 & 16 & 0.0556 \\
\hline 27 & 0.0010 & 0.0010 & 0.95 & 32 & 0.0417 \\
\hline 28 & 0.0050 & 0.0001 & 0.85 & 8 & 0.0556 \\
\hline 29 & 0.0050 & 0.0001 & 0.85 & 16 & 0.0486 \\
\hline 30 & 0.0050 & 0.0001 & 0.85 & 32 & 0.0625 \\
\hline 31 & 0.0050 & 0.0001 & 0.90 & 8 & 0.0625 \\
\hline 32 & 0.0050 & 0.0001 & 0.90 & 16 & 0.0556 \\
\hline 33 & 0.0050 & 0.0001 & 0.90 & 32 & 0.0625 \\
\hline 34 & 0.0050 & 0.0001 & 0.95 & 8 & 0.1319 \\
\hline 35 & 0.0050 & 0.0001 & 0.95 & 16 & 0.0764 \\
\hline 36 & 0.0050 & 0.0001 & 0.95 & 32 & 0.0417 \\
\hline 37 & 0.0050 & 0.0050 & 0.85 & 8 & 0.0417 \\
\hline 38 & 0.0050 & 0.0050 & 0.85 & 16 & 0.0556 \\
\hline 39 & 0.0050 & 0.0050 & 0.85 & 32 & 0.0694 \\
\hline 40 & 0.0050 & 0.0050 & 0.90 & 8 & 0.0417 \\
\hline 41 & 0.0050 & 0.0050 & 0.90 & 16 & 0.8194 \\
\hline 42 & 0.0050 & 0.0050 & 0.90 & 32 & 0.0556 \\
\hline 43 & 0.0050 & 0.0050 & 0.95 & 8 & 0.0833 \\
\hline 44 & 0.0050 & 0.0050 & 0.95 & 16 & 0.0556 \\
\hline 45 & 0.0050 & 0.0050 & 0.95 & 32 & 0.0486 \\
\hline 46 & 0.0050 & 0.0010 & 0.85 & 8 & 0.0417 \\
\hline 47 & 0.0050 & 0.0010 & 0.85 & 16 & 0.0625 \\
\hline 48 & 0.0050 & 0.0010 & 0.85 & 32 & 0.0486 \\
\hline 49 & 0.0050 & 0.0010 & 0.90 & 8 & 0.0694 \\
\hline 50 & 0.0050 & 0.0010 & 0.90 & 16 & 0.0486 \\
\hline 51 & 0.0050 & 0.0010 & 0.90 & 32 & 0.0556 \\
\hline 52 & 0.0050 & 0.0010 & 0.95 & 8 & 0.1458 \\
\hline 53 & 0.0050 & 0.0010 & 0.95 & 16 & 0.0625 \\
\hline 54 & 0.0050 & 0.0010 & 0.95 & 32 & 0.0556 \\
\hline 55 & 0.0100 & 0.0001 & 0.85 & 8 & 0.0556 \\
\hline 56 & 0.0100 & 0.0001 & 0.85 & 16 & 0.0417 \\
\hline 57 & 0.0100 & 0.0001 & 0.85 & 32 & 0.0556 \\
\hline 58 & 0.0100 & 0.0001 & 0.90 & 8 & 0.1458 \\
\hline 59 & 0.0100 & 0.0001 & 0.90 & 16 & 0.0625 \\
\hline 60 & 0.0100 & 0.0001 & 0.90 & 32 & 0.0347 \\
\hline 61 & 0.0100 & 0.0001 & 0.95 & 8 & 0.0903 \\
\hline 62 & 0.0100 & 0.0001 & 0.95 & 16 & 0.1111 \\
\hline 63 & 0.0100 & 0.0001 & 0.95 & 32 & 0.0556 \\
\hline 64 & 0.0100 & 0.0050 & 0.85 & 8 & 0.8194 \\
\hline 65 & 0.0100 & 0.0050 & 0.85 & 16 & 0.0208 \\
\hline 66 & 0.0100 & 0.0050 & 0.85 & 32 & 0.0556 \\
\hline 67 & 0.0100 & 0.0050 & 0.90 & 8 & 0.1319 \\
\hline 68 & 0.0100 & 0.0050 & 0.90 & 16 & 0.8194 \\
\hline 69 & 0.0100 & 0.0050 & 0.90 & 32 & 0.0833 \\
\hline 70 & 0.0100 & 0.0050 & 0.95 & 8 & 0.1597 \\
\hline 71 & 0.0100 & 0.0050 & 0.95 & 16 & 0.0625 \\
\hline 72 & 0.0100 & 0.0050 & 0.95 & 32 & 0.0764 \\
\hline 73 & 0.0100 & 0.0010 & 0.85 & 8 & 0.1111 \\
\hline 74 & 0.0100 & 0.0010 & 0.85 & 16 & 0.0556 \\
\hline 75 & 0.0100 & 0.0010 & 0.85 & 32 & 0.0486 \\
\hline 76 & 0.0100 & 0.0010 & 0.90 & 8 & 0.0972 \\
\hline 77 & 0.0100 & 0.0010 & 0.90 & 16 & 0.0417 \\
\hline 78 & 0.0100 & 0.0010 & 0.90 & 32 & 0.0556 \\
\hline 79 & 0.0100 & 0.0010 & 0.95 & 8 & 0.1875 \\
\hline 80 & 0.0100 & 0.0010 & 0.95 & 16 & 0.1389 \\
\hline 81 & 0.0100 & 0.0010 & 0.95 & 32 & 0.0556 \\
\hline
\end{tabular}


Table 10: Hyperparameters and fval values for the GS and ResNet50 aided method

\begin{tabular}{|c|c|c|c|c|c|}
\hline Run & ILR & $\ell_{2} \mathbf{R}$ & Mom & MiB & fval \\
\hline 1 & 0.0010 & 0.0001 & 0.85 & 8 & 0.0972 \\
\hline 2 & 0.0010 & 0.0001 & 0.85 & 16 & 0.0625 \\
\hline 3 & 0.0010 & 0.0001 & 0.85 & 32 & 0.0556 \\
\hline 4 & 0.0010 & 0.0001 & 0.90 & 8 & 0.8194 \\
\hline 5 & 0.0010 & 0.0001 & 0.90 & 16 & 0.0486 \\
\hline 6 & 0.0010 & 0.0001 & 0.90 & 32 & 0.0625 \\
\hline 7 & 0.0010 & 0.0001 & 0.95 & 8 & 0.0486 \\
\hline 8 & 0.0010 & 0.0001 & 0.95 & 16 & 0.0694 \\
\hline 9 & 0.0010 & 0.0001 & 0.95 & 32 & 0.0625 \\
\hline 10 & 0.0010 & 0.0050 & 0.85 & 8 & 0.0694 \\
\hline 11 & 0.0010 & 0.0050 & 0.85 & 16 & 0.0694 \\
\hline 12 & 0.0010 & 0.0050 & 0.85 & 32 & 0.0764 \\
\hline 13 & 0.0010 & 0.0050 & 0.90 & 8 & 0.0486 \\
\hline 14 & 0.0010 & 0.0050 & 0.90 & 16 & 0.0417 \\
\hline 15 & 0.0010 & 0.0050 & 0.90 & 32 & 0.0694 \\
\hline 16 & 0.0010 & 0.0050 & 0.95 & 8 & 0.0625 \\
\hline 17 & 0.0010 & 0.0050 & 0.95 & 16 & 0.0556 \\
\hline 18 & 0.0010 & 0.0050 & 0.95 & 32 & 0.0764 \\
\hline 19 & 0.0010 & 0.0010 & 0.85 & 8 & 0.0833 \\
\hline 20 & 0.0010 & 0.0010 & 0.85 & 16 & 0.0694 \\
\hline 21 & 0.0010 & 0.0010 & 0.85 & 32 & 0.0625 \\
\hline 22 & 0.0010 & 0.0010 & 0.90 & 8 & 0.0556 \\
\hline 23 & 0.0010 & 0.0010 & 0.90 & 16 & 0.0625 \\
\hline 24 & 0.0010 & 0.0010 & 0.90 & 32 & 0.0903 \\
\hline 25 & 0.0010 & 0.0010 & 0.95 & 8 & 0.0486 \\
\hline 26 & 0.0010 & 0.0010 & 0.95 & 16 & 0.0694 \\
\hline 27 & 0.0010 & 0.0010 & 0.95 & 32 & 0.0486 \\
\hline 28 & 0.0050 & 0.0001 & 0.85 & 8 & 0.0347 \\
\hline 29 & 0.0050 & 0.0001 & 0.85 & 16 & 0.0278 \\
\hline 30 & 0.0050 & 0.0001 & 0.85 & 32 & 0.0625 \\
\hline 31 & 0.0050 & 0.0001 & 0.90 & 8 & 0.0486 \\
\hline 32 & 0.0050 & 0.0001 & 0.90 & 16 & 0.0625 \\
\hline 33 & 0.0050 & 0.0001 & 0.90 & 32 & 0.0694 \\
\hline 34 & 0.0050 & 0.0001 & 0.95 & 8 & 0.0764 \\
\hline 35 & 0.0050 & 0.0001 & 0.95 & 16 & 0.0694 \\
\hline 36 & 0.0050 & 0.0001 & 0.95 & 32 & 0.0556 \\
\hline 37 & 0.0050 & 0.0050 & 0.85 & 8 & 0.0417 \\
\hline 38 & 0.0050 & 0.0050 & 0.85 & 16 & 0.0417 \\
\hline 39 & 0.0050 & 0.0050 & 0.85 & 32 & 0.0764 \\
\hline 40 & 0.0050 & 0.0050 & 0.90 & 8 & 0.0417 \\
\hline 41 & 0.0050 & 0.0050 & 0.90 & 16 & 0.0625 \\
\hline 42 & 0.0050 & 0.0050 & 0.90 & 32 & 0.0625 \\
\hline 43 & 0.0050 & 0.0050 & 0.95 & 8 & 0.0694 \\
\hline 44 & 0.0050 & 0.0050 & 0.95 & 16 & 0.0764 \\
\hline 45 & 0.0050 & 0.0050 & 0.95 & 32 & 0.0417 \\
\hline 46 & 0.0050 & 0.0010 & 0.85 & 8 & 0.0486 \\
\hline 47 & 0.0050 & 0.0010 & 0.85 & 16 & 0.0486 \\
\hline 48 & 0.0050 & 0.0010 & 0.85 & 32 & 0.0625 \\
\hline 49 & 0.0050 & 0.0010 & 0.90 & 8 & 0.0278 \\
\hline 50 & 0.0050 & 0.0010 & 0.90 & 16 & 0.0486 \\
\hline 51 & 0.0050 & 0.0010 & 0.90 & 32 & 0.0486 \\
\hline 52 & 0.0050 & 0.0010 & 0.95 & 8 & 0.0833 \\
\hline 53 & 0.0050 & 0.0010 & 0.95 & 16 & 0.0486 \\
\hline 54 & 0.0050 & 0.0010 & 0.95 & 32 & 0.0347 \\
\hline 55 & 0.0100 & 0.0001 & 0.85 & 8 & 0.0417 \\
\hline 56 & 0.0100 & 0.0001 & 0.85 & 16 & 0.0486 \\
\hline 57 & 0.0100 & 0.0001 & 0.85 & 32 & 0.0486 \\
\hline 58 & 0.0100 & 0.0001 & 0.90 & 8 & 0.8194 \\
\hline 59 & 0.0100 & 0.0001 & 0.90 & 16 & 0.0833 \\
\hline 60 & 0.0100 & 0.0001 & 0.90 & 32 & 0.0625 \\
\hline 61 & 0.0100 & 0.0001 & 0.95 & 8 & 0.1875 \\
\hline 62 & 0.0100 & 0.0001 & 0.95 & 16 & 0.1667 \\
\hline 63 & 0.0100 & 0.0001 & 0.95 & 32 & 0.0486 \\
\hline 64 & 0.0100 & 0.0050 & 0.85 & 8 & 0.0347 \\
\hline 65 & 0.0100 & 0.0050 & 0.85 & 16 & 0.0417 \\
\hline 66 & 0.0100 & 0.0050 & 0.85 & 32 & 0.0486 \\
\hline 67 & 0.0100 & 0.0050 & 0.90 & 8 & 0.1597 \\
\hline 68 & 0.0100 & 0.0050 & 0.90 & 16 & 0.0486 \\
\hline 69 & 0.0100 & 0.0050 & 0.90 & 32 & 0.0694 \\
\hline 70 & 0.0100 & 0.0050 & 0.95 & 8 & 0.8194 \\
\hline 71 & 0.0100 & 0.0050 & 0.95 & 16 & 0.0625 \\
\hline 72 & 0.0100 & 0.0050 & 0.95 & 32 & 0.0972 \\
\hline 73 & 0.0100 & 0.0010 & 0.85 & 8 & 0.0694 \\
\hline 74 & 0.0100 & 0.0010 & 0.85 & 16 & 0.8194 \\
\hline 75 & 0.0100 & 0.0010 & 0.85 & 32 & 0.0556 \\
\hline 76 & 0.0100 & 0.0010 & 0.90 & 8 & 0.0417 \\
\hline 77 & 0.0100 & 0.0010 & 0.90 & 16 & 0.0347 \\
\hline 78 & 0.0100 & 0.0010 & 0.90 & 32 & 0.0903 \\
\hline 79 & 0.0100 & 0.0010 & 0.95 & 8 & 0.1111 \\
\hline 80 & 0.0100 & 0.0010 & 0.95 & 16 & 0.0833 \\
\hline 81 & 0.0100 & 0.0010 & 0.95 & 32 & 0.0694 \\
\hline
\end{tabular}

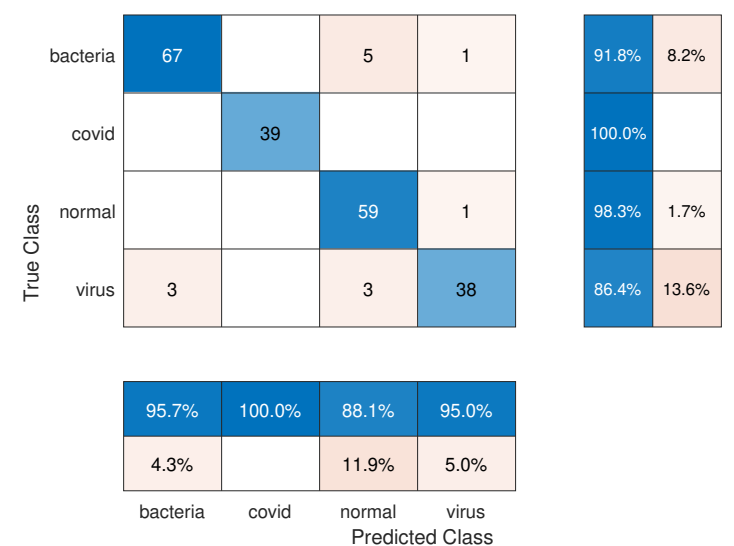

Fig. 8: The confusion matrix for GoogleNet on test data
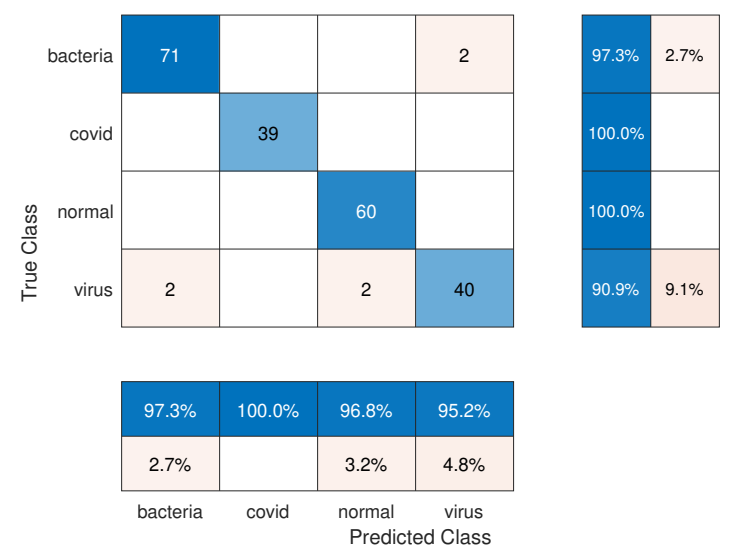

Fig. 9: The confusion matrix for ResNet18 on test data
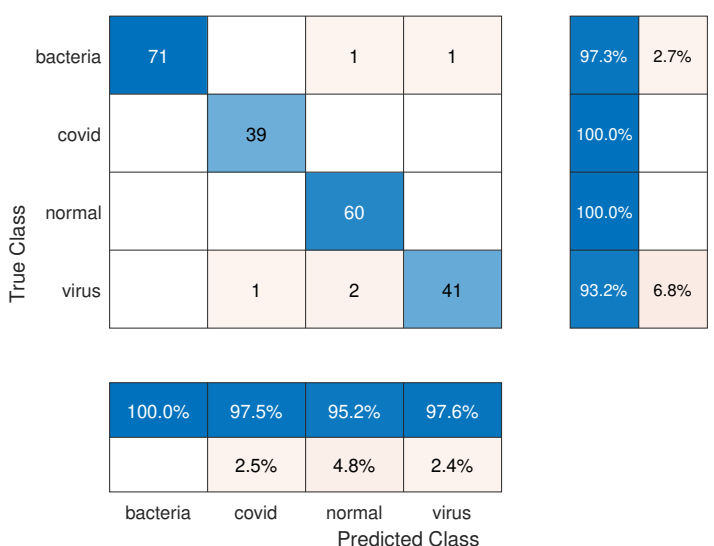

Fig. 10: The confusion matrix for ResNet50 on test data

The confusion matrices which are formed according to the accuracy performance of the proposed methods are presented in Fig. 8 - 10. In addition, the training progress graphics for the final training processes of the proposed methods are given in Fig. 11 - 13, respectively. 


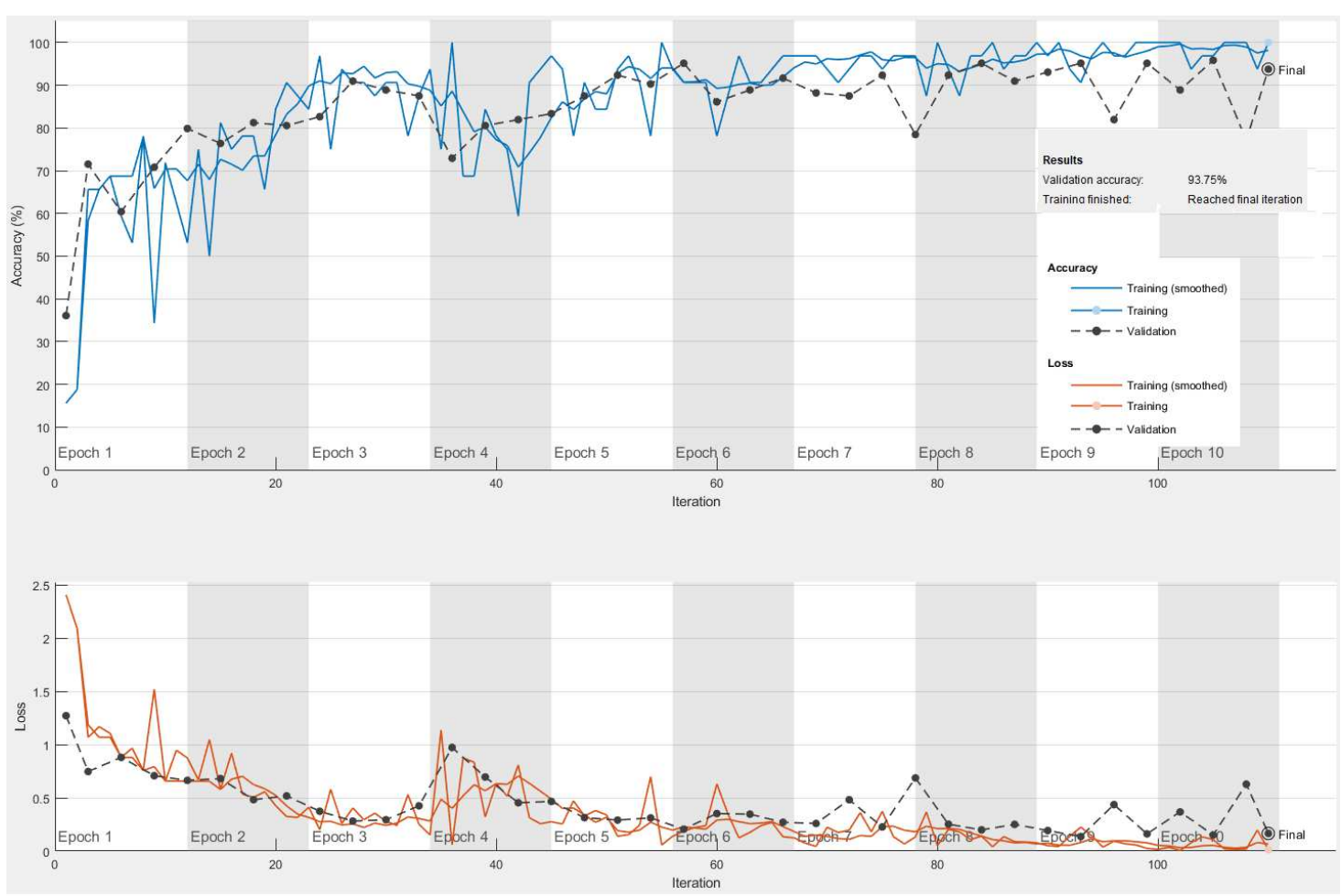

Fig. 11: Training progress for GoogleNet aided method

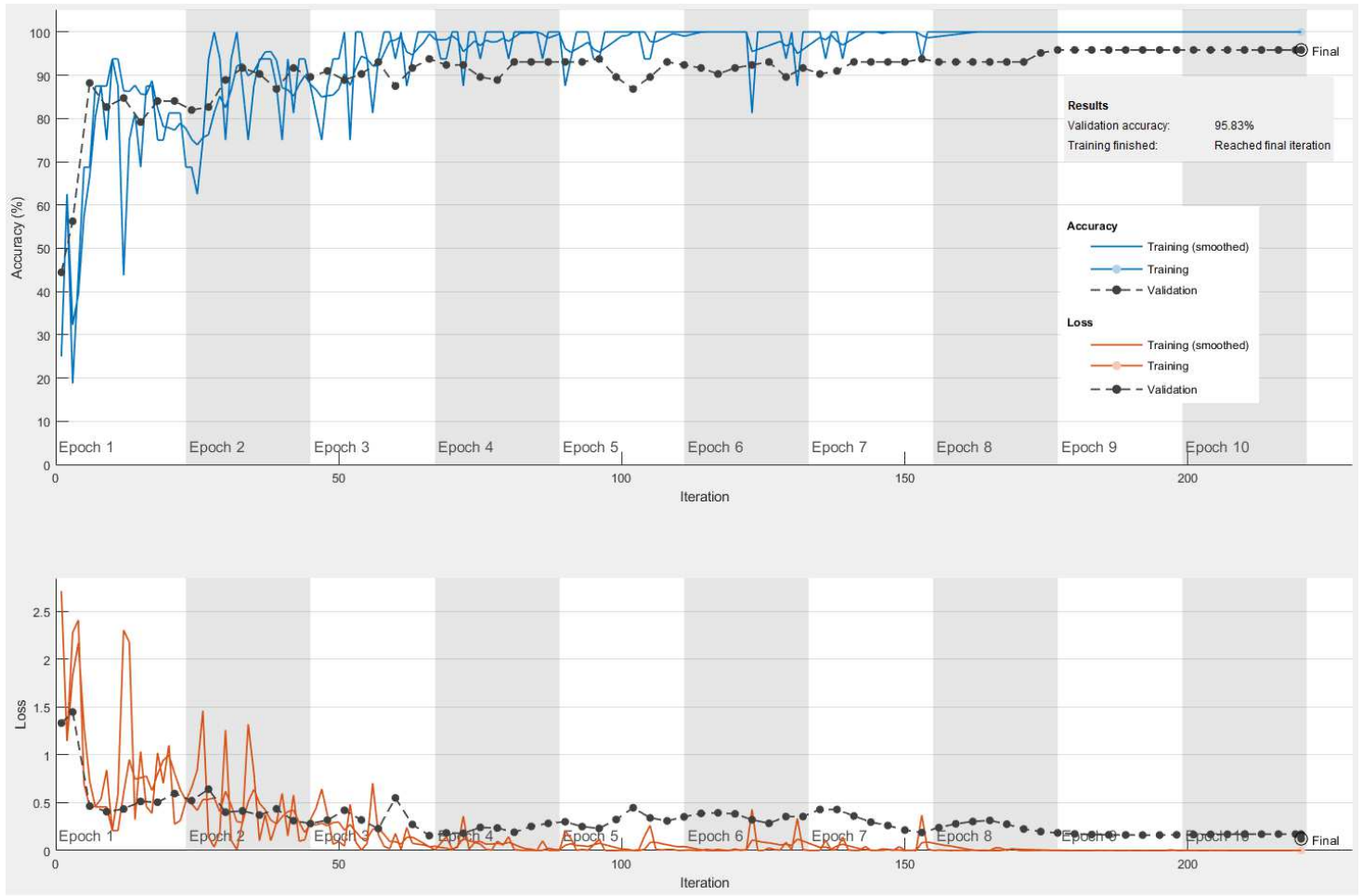

Fig. 12: Training progress for ResNet18 aided method 


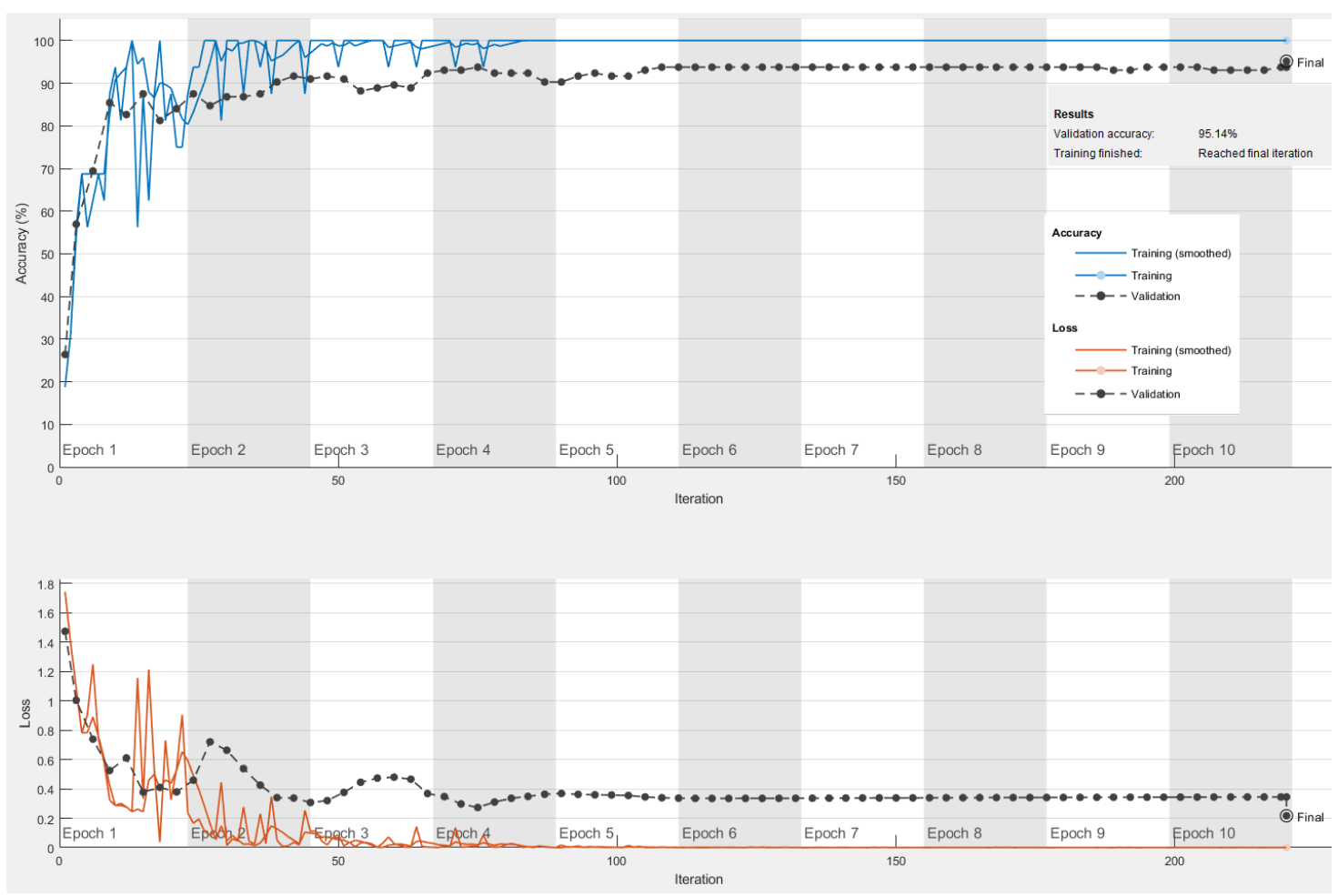

Fig. 13: Training progress for ResNet50 aided method

\section{Conclusion}

COVID-19 is a deadly virus that currently impacting the entire world. The early detection of this virus provides both a slowing down of the outbreak and the recovery of the daignosed patients more quickly. Recently, deep learning methods are frequently used in classification problems. Additionally, the performance of deep learning methods is implemented by a hyperparameter selection. In this study, a classification system was developed by using both the GS and pre-trained CNN models. Here, while GS was used for hyperparameter optimization, the pre-trained models were used for transfer learning. With the proposed method, classification experiments were carried for 4 different classes on X-Ray images of the lungs. These categories of the dataset include bacteria, COVID-19, normal, and virus. In the proposed method, 3 different pre-trained models (GoogleNet, ResNet18, ResNet50) were used. The highest accuracy rate $(97.69 \%)$ was obtained with the GS and ResNet50 aided proposed method.

In future studies, different pre-trained models will be used for transfer learning. Additionally, different global search methods would be employed for hyperparameter optimization.

\section{Conflict of interest}

The author declares that there is no conflict of interests regarding the publication of this article. Tayyip Ozcan received no financial support for the research, authorship, and/or publication of this article.

\section{References}

1. Akcay, S., Kundegorski, M., Willcocks, C., Breckon, T.: Using deep convolutional neural network architectures for object classification and detection within x-ray baggage security imagery. IEEE Transactions on Information Forensics and Security 13(9), 2203-2215 (2018)

2. Alnujaim, I., Alali, H., Khan, F., Kim, Y.: Hand gesture recognition using input impedance variation of two antennas with transfer learning. IEEE Sensors Journal 18(10), 4129-4135 (2018)

3. Apostolopoulos, I.D., Bessiana, T.: Covid-19: Automatic detection from x-ray images utilizing transfer learning with convolutional neural networks. arXiv preprint arXiv:2003.11617 (2020)

4. Bussey, D., Glandon, A., Vidyaratne, L., Alam, M., Iftekharuddin, K.M.: Convolutional neural network transfer learning for robust face recognition in nao humanoid robot. In: 2017 IEEE Symposium Series on Computational Intelligence (SSCI), pp. 1-7. IEEE, Honolulu (2017). DOI 10.1109/SSCI.2017.8285347

5. Cohen, J.P., Morrison, P., Dao, L.: Covid-19 image data collection. arXiv 2003.11597 (2020). URL https:// github.com/ieee8023/covid-chestxray-dataset 
6. Cote-Allard, U., Fall, C.L., Campeau-Lecours, A., Gosselin, C., Laviolette, F., Gosselin, B.: Transfer learning for sEMG hand gestures recognition using convolutional neural networks. In: 2017 IEEE International Conference on Systems, Man, and Cybernetics (SMC), pp. 1663-1668 (2017)

7. Czuszynski, K., Ruminski, J., Kwasniewska, A.: Gesture recognition with the linear optical sensor and recurrent neural networks. IEEE Sensors Journal 18(13), 54295438 (2018)

8. Desmet, B., Hoste, V.: Online suicide prevention through optimised text classification. Information Sciences 439440, 61-78 (2018)

9. Diaz, G., Fokoue, A., Nannicini, G., Samulowitz, H.: An effective algorithm for hyperparameter optimization of neural networks. arXiv 61(4), 1-20 (2017)

10. El-Din Hemdan, E., Shouman, M.A., Karar, M.E.: Covidx-net: A framework of deep learning classifiers to diagnose covid-19 in x-ray images. arXiv pp. arXiv-2003 (2020)

11. Ghoshal, B., Tucker, A.: Estimating uncertainty and interpretability in deep learning for coronavirus (covid19) detection. arXiv preprint arXiv:2003.10769 (2020)

12. Han, D., Liu, Q., Fan, W.: A new image classification method using CNN transfer learning and web data augmentation. Expert Systems with Applications 95, 4356 (2018)

13. He, K., Zhang, X., Ren, S., Sun, J.: Deep residual learning for image recognition. In: Proceedings of the IEEE conference on computer vision and pattern recognition, pp. 770-778. IEEE, Las Vegas (2016)

14. Hinz, T., Navarro-Guerrero, N., Magg, S., Wermter, S.: Speeding up the Hyperparameter Optimization of Deep Convolutional Neural Networks. International Journal of Computational Intelligence and Applications 17(02), $1-15(2018)$

15. KARCIOĞLU, Ö.: What is coronaviruses, and how can we protect ourselves? Anka Tıp Dergisi 2(1), 66-71

16. Kilic, E., Ozturk, S.: A subclass supported convolutional neural network for object detection and localization in remote-sensing images. International Journal of Remote Sensing 40(11), 4193-4212 (2019)

17. Klein, A., Falkner, S., Bartels, S., Hennig, P., Hutter, F.: Fast Bayesian Optimization of Machine Learning Hyperparameters on Large Datasets. arXiv 54, 1-9 (2016)

18. Krizhevsky, A., Sutskever, I., Hinton, G.: Imagenet classification with deep convolutional neural networks. NIPS 25, 1106-1114 (2012)

19. Li, G., Tang, H., Sun, Y., Kong, J., Jiang, G., Jiang, D., Tao, B., Xu, S., Liu, H.: Hand gesture recognition based on convolution neural network. Cluster Computing 22(2), 2719-2729 (2019)

20. Mathworks: Documentation. https://www.mathworks. com/help. Online; accessed 30 March 2020

21. Narin, A., Kaya, C., Pamuk, Z.: Automatic detection of coronavirus disease (covid-19) using x-ray images and deep convolutional neural networks. arXiv preprint arXiv:2003.10849 (2020)

22. Ozcan, T., Basturk, A.: Lip reading using convolutional neural networks with and without pre-trained models. Balkan Journal of Electrical and Computer Engineering 7(2), 195-201 (2019)

23. Ozcan, T., Basturk, A.: Static image-based emotion recognition using convolutional neural network. In: 2019 27th Signal Processing and Communications
Applications Conference (SIU), pp. 1-4 (2019). DOI 10.1109/SIU.2019.8806408

24. Ozcan, T., Basturk, A.: Transfer learning-based convolutional neural networks with heuristic optimization for hand gesture recognition. Neural Computing and Applications (2019). DOI 10.1007/s00521-019-04427-y

25. Ozcan, T., Basturk, A.: Human action recognition with deep learning and structural optimization using a hybrid heuristic algorithm. Cluster Computing pp. 1-14 (2020)

26. Ozcan, T., Basturk, A.: Performance improvement of pretrained convolutional neural networks for action recognition. The Computer Journal pp. 1-12 (2020)

27. Paul Mooney: Chest X-Ray Images (Pneumonia). https://www.kaggle.com/paultimothymooney/ chest-xray-pneumonia. Online; accessed 20 March 2020

28. Rahnama, A.H.A., Toloo, M., Zaidenberg, N.J.: An LPbased hyperparameter optimization model for language modeling. arXiv pp. 1-11 (2018)

29. Riegler, M.: HINDSIGHT: An R-based Framework Towards Long Short Term Memory (LSTM) Optimization. In: Proceedings of the 9th ACM Multimedia Systems Conference, pp. 381-386 (2018)

30. Rubio, G., Pomares, H., Rojas, I., Herrera, L.J.: A heuristic method for parameter selection in LS-SVM: Application to time series prediction. International Journal of Forecasting 27(3), 725-739 (2011)

31. Sánchez-Illana, Á., Pérez-Guaita, D., Cuesta-García, D., Sanjuan-Herráez, J.D., Vento, M., Ruiz-Cerdá, J.L., Quintás, G., Kuligowski, J.: Model selection for withinbatch effect correction in UPLC-MS metabolomics using quality control - Support vector regression. Analytica Chimica Acta 1026, 62-68 (2018)

32. Sethy, P.K., Behera, S.K.: Detection of coronavirus disease (covid-19) based on deep features (2020)

33. Società Italiana di Radiologia Medica e Interventistica: COVID-19 Database. https://www.sirm.org. Online; accessed 28 March 2020

34. Szegedy, C., Liu, W., Jia, Y., Sermanet, P., Reed, S., Anguelov, D., Erhan, D., Vanhoucke, V., Rabinovich, A.: Going deeper with convolutions. In: Proceedings of the IEEE conference on computer vision and pattern recognition, pp. 1-9. IEEE, Boston (2015)

35. Tang, Y., Wu, B., Peng, L., Liu, C.: Semi-supervised transfer learning for convolutional neural network based chinese character recognition. In: 2017 14th IAPR International Conference on Document Analysis and Recognition (ICDAR), pp. 441-447. CPS, Kyoto (2017). DOI 10.1109/ICDAR.2017.79

36. Wang, L., Wong, A.: Covid-net: A tailored deep convolutional neural network design for detection of covid-19 cases from chest radiography images. arXiv preprint arXiv:2003.09871 (2020)

37. Xia, Y., Huang, D., Wang, Y.: Detecting smiles of young children via deep transfer learning. In: 2017 IEEE International Conference on Computer Vision Workshops (ICCVW), pp. 1673-1681. Italy, Venice (2017). DOI 10.1109/ICCVW.2017.196

38. Yao, C., Cai, D., Bu, J., Chen, G.: Pre-training the deep generative models with adaptive hyperparameter optimization. Neurocomputing 247, 144-155 (2017)

39. Zhang, J., Xie, Y., Li, Y., Shen, C., Xia, Y.: Covid-19 screening on chest $\mathrm{X}$-ray images using deep learning based anomaly detection. arXiv preprint arXiv:2003.12338 (2020) 
Figures

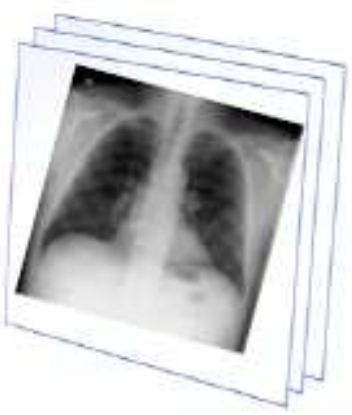

Input

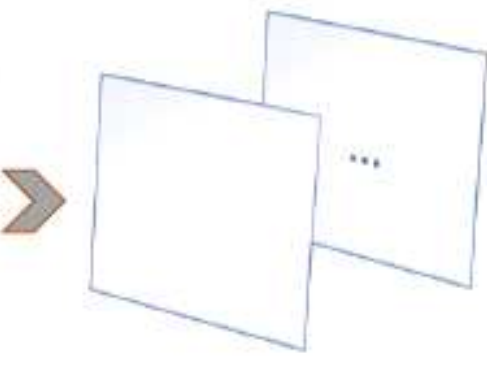

Convolution

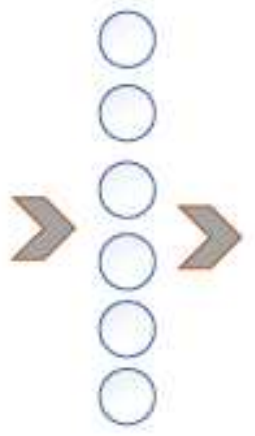

Pooling Fully Connected
Bacteria

Covid

Normal

Virus

Output

\section{Figure 1}

The main layers of a CNN architecture

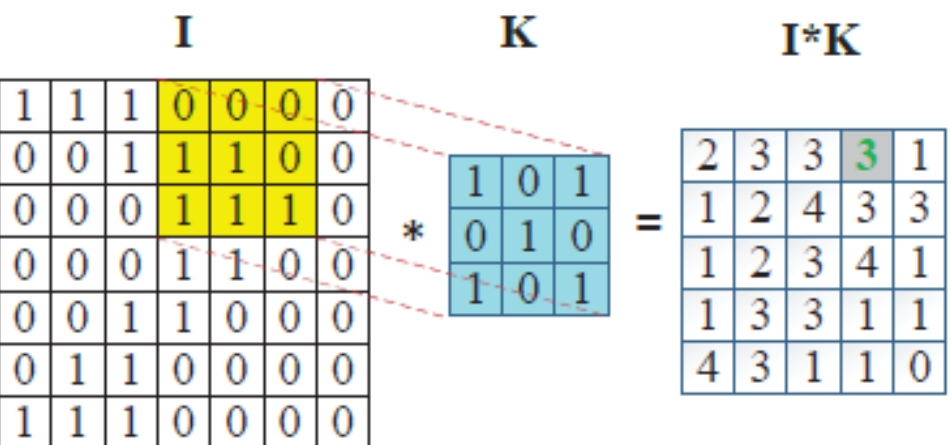

Figure 2

An example of the convolution operation.

\begin{tabular}{|c|c|c|c|c|c|c|}
\hline 11 & 12 & 11 & 15 & & & \\
\hline 7 & 4 & 12 & 9 & $2 \times 2$ Max-Pool & 12 & 15 \\
\hline 3 & 15 & 25 & 15 & & 18 & 25 \\
\hline 18 & 13 & 4 & 12 & & & \\
\hline
\end{tabular}

Figure 3

An example of the maxpooling operation. 


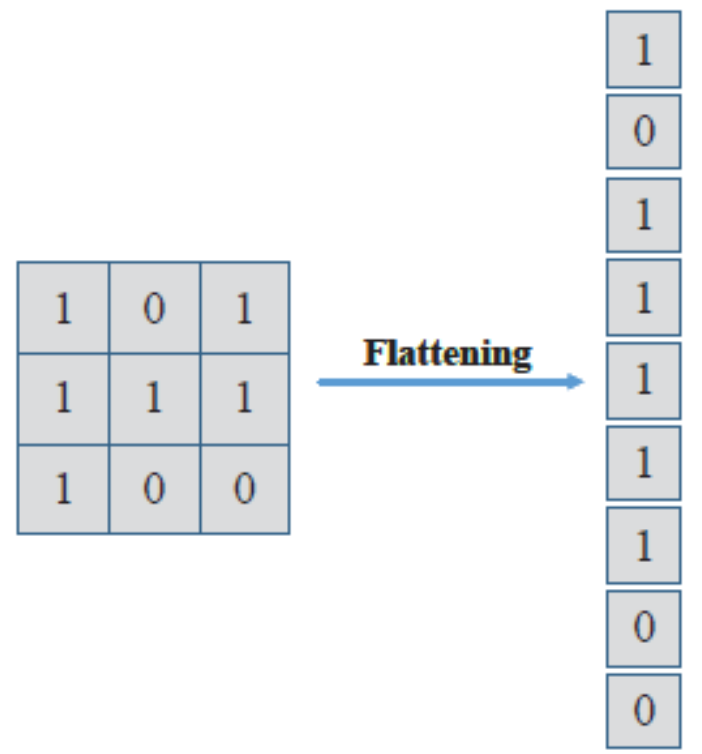

Figure 4

An example of the attening operation.

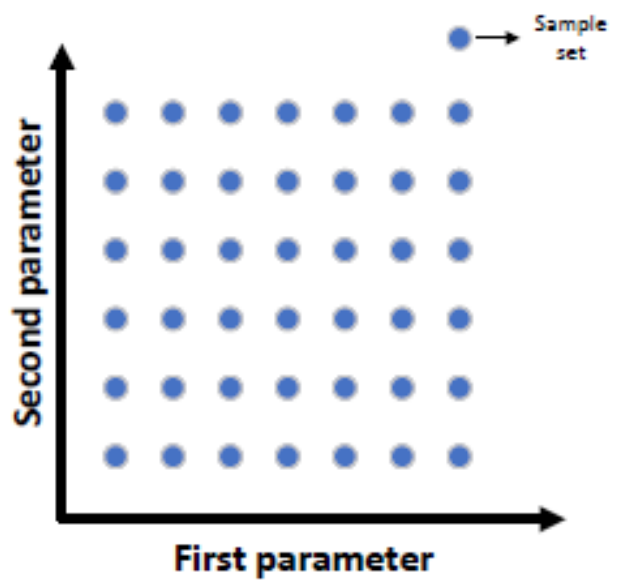

Figure 5

Parameter sets in the search space for GS 


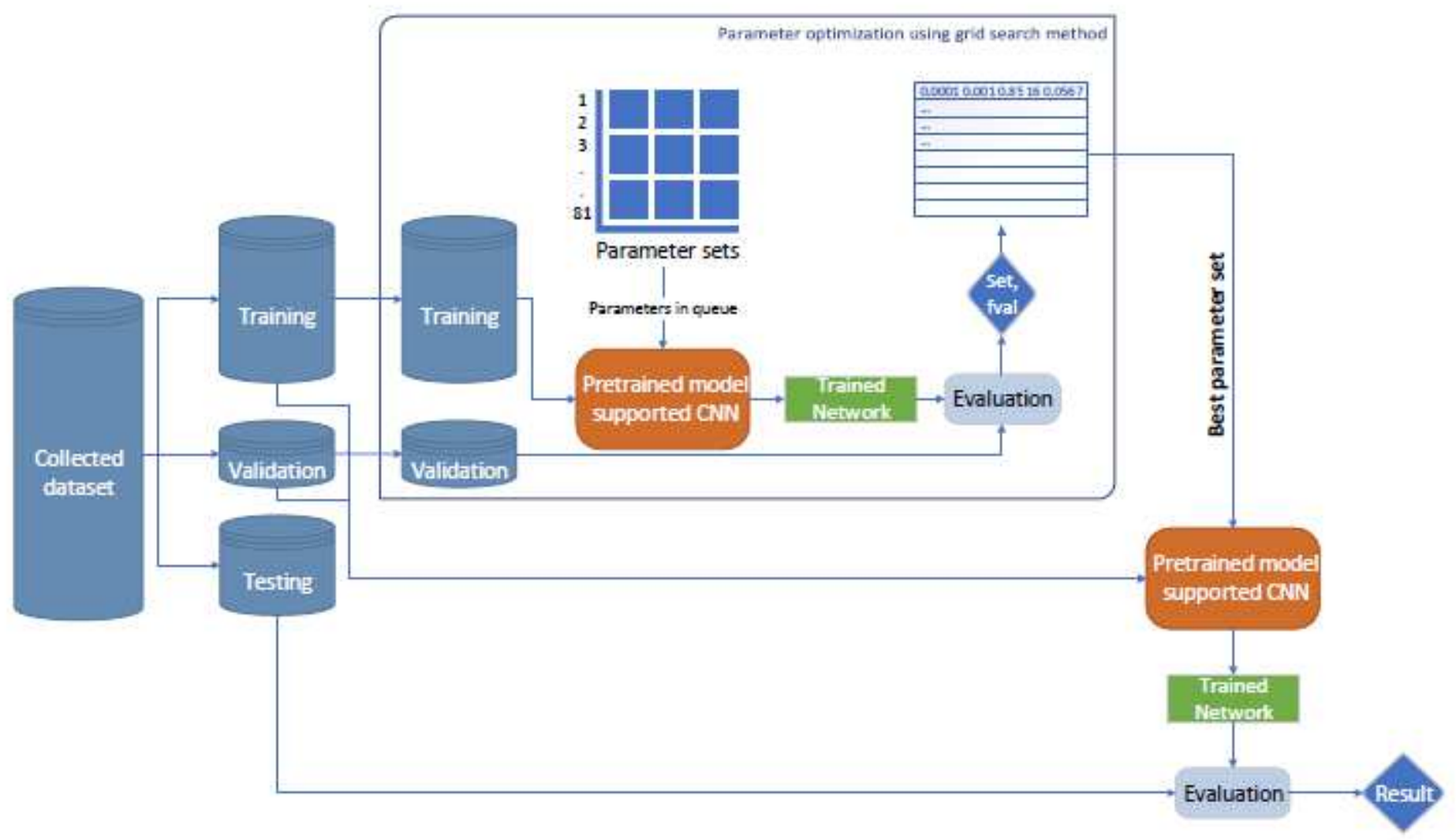

Figure 6

The GS and Pre-trained model aided method

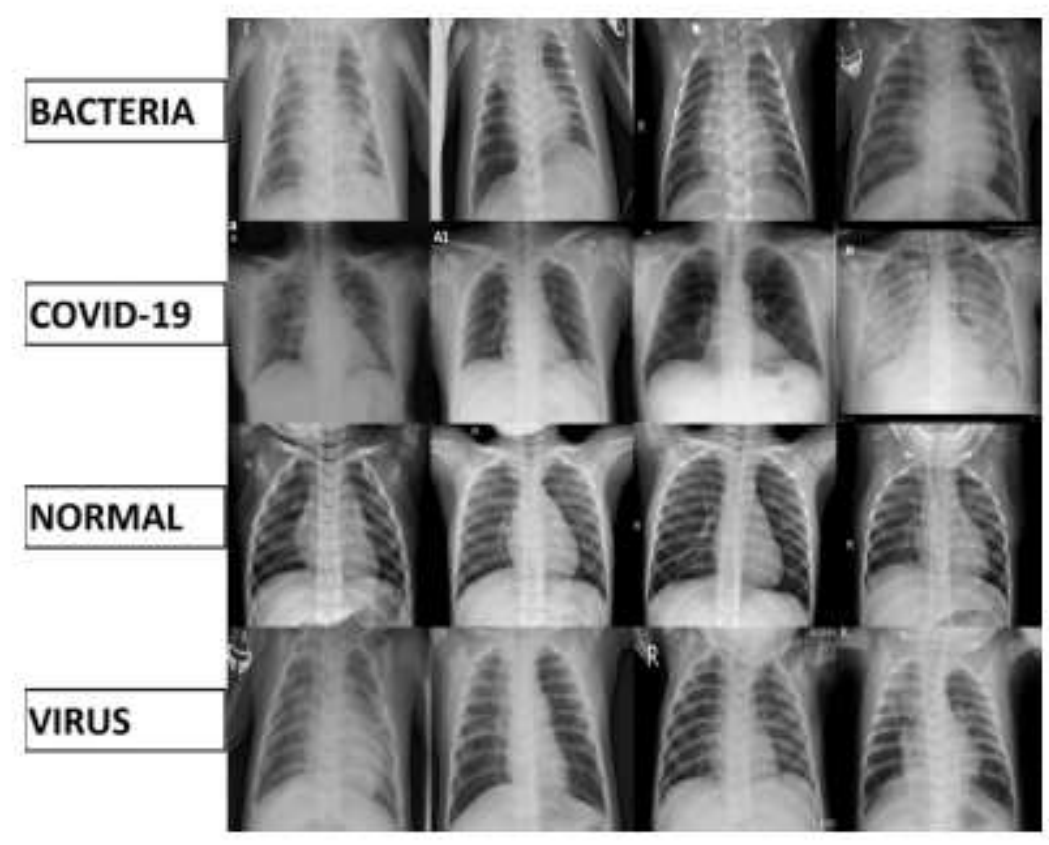

Figure 7 
Sample images from collected dataset
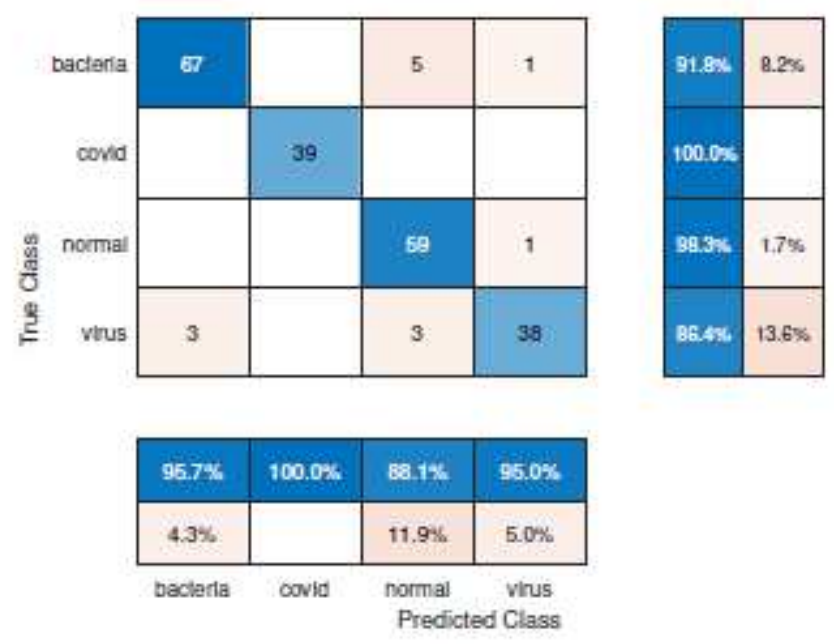

Figure 8

The confusion matrix for GoogleNet on test data
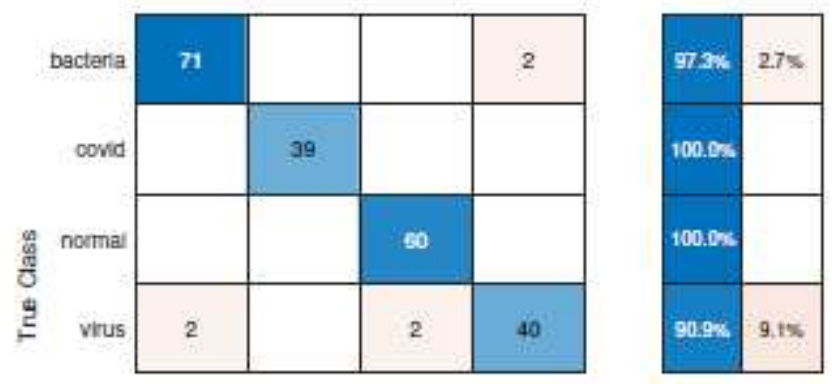

\begin{tabular}{|c|c|c|c|}
\hline $97.3 \%$ & $100.0 \%$ & $86.2 \%$ & 85.24 \\
\hline $2.7 \%$ & & $3.2 \%$ & $4.8 \%$ \\
\hline Daclarla $\quad$ covid & \multicolumn{3}{|c|}{$\begin{array}{c}\text { normal } \\
\text { Predicted Class }\end{array}$} \\
\hline
\end{tabular}

\section{Figure 9}

The confusion matrix for ResNet18 on test data 

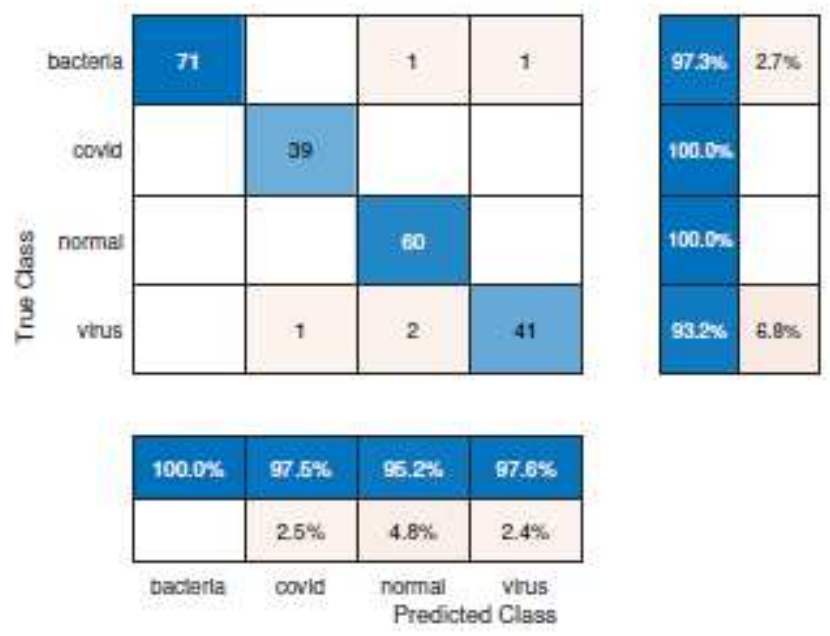

\section{Figure 10}

The confusion matrix for ResNet50 on test data
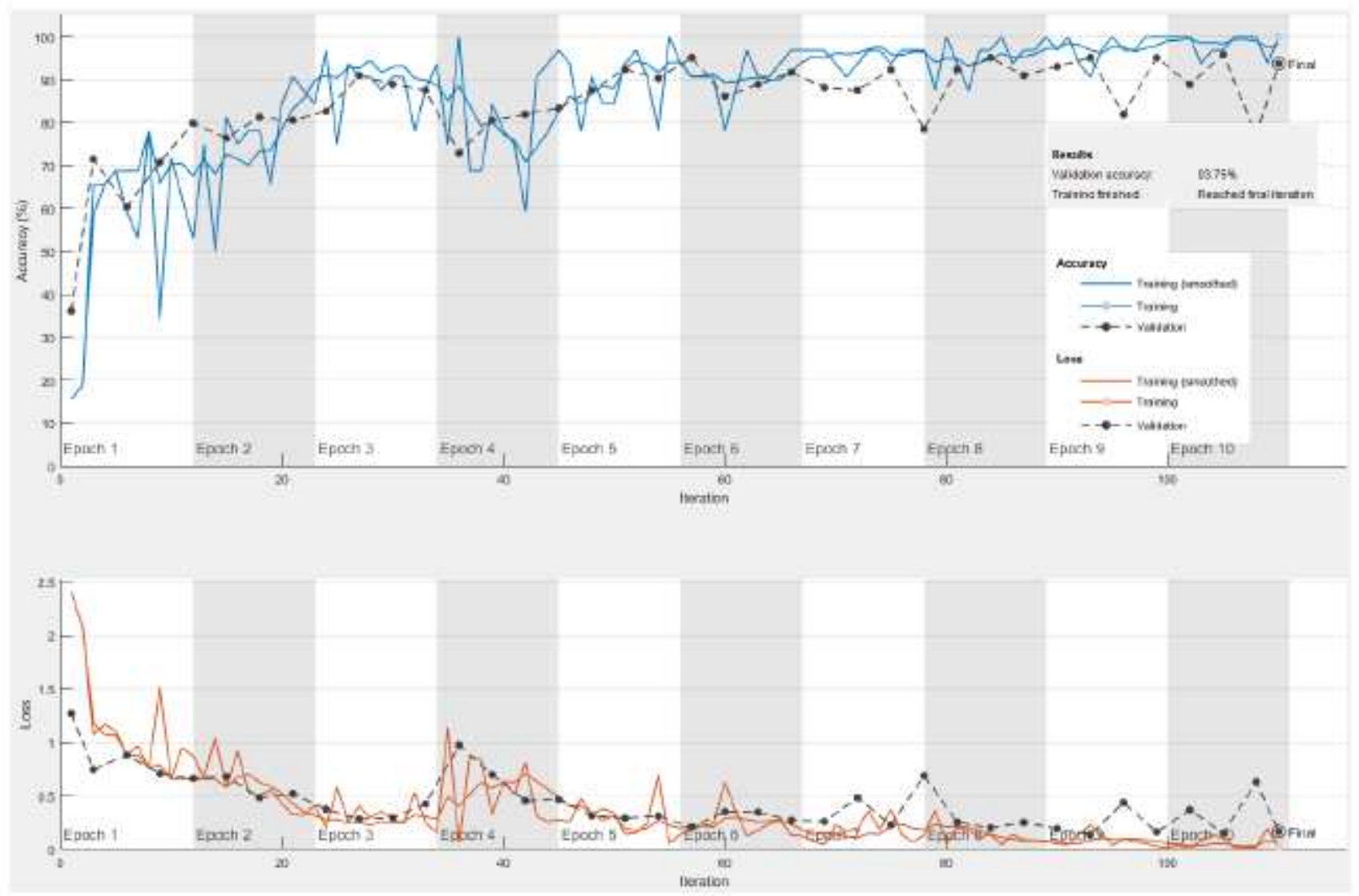

Figure 11

Training progress for GoogleNet aided method 


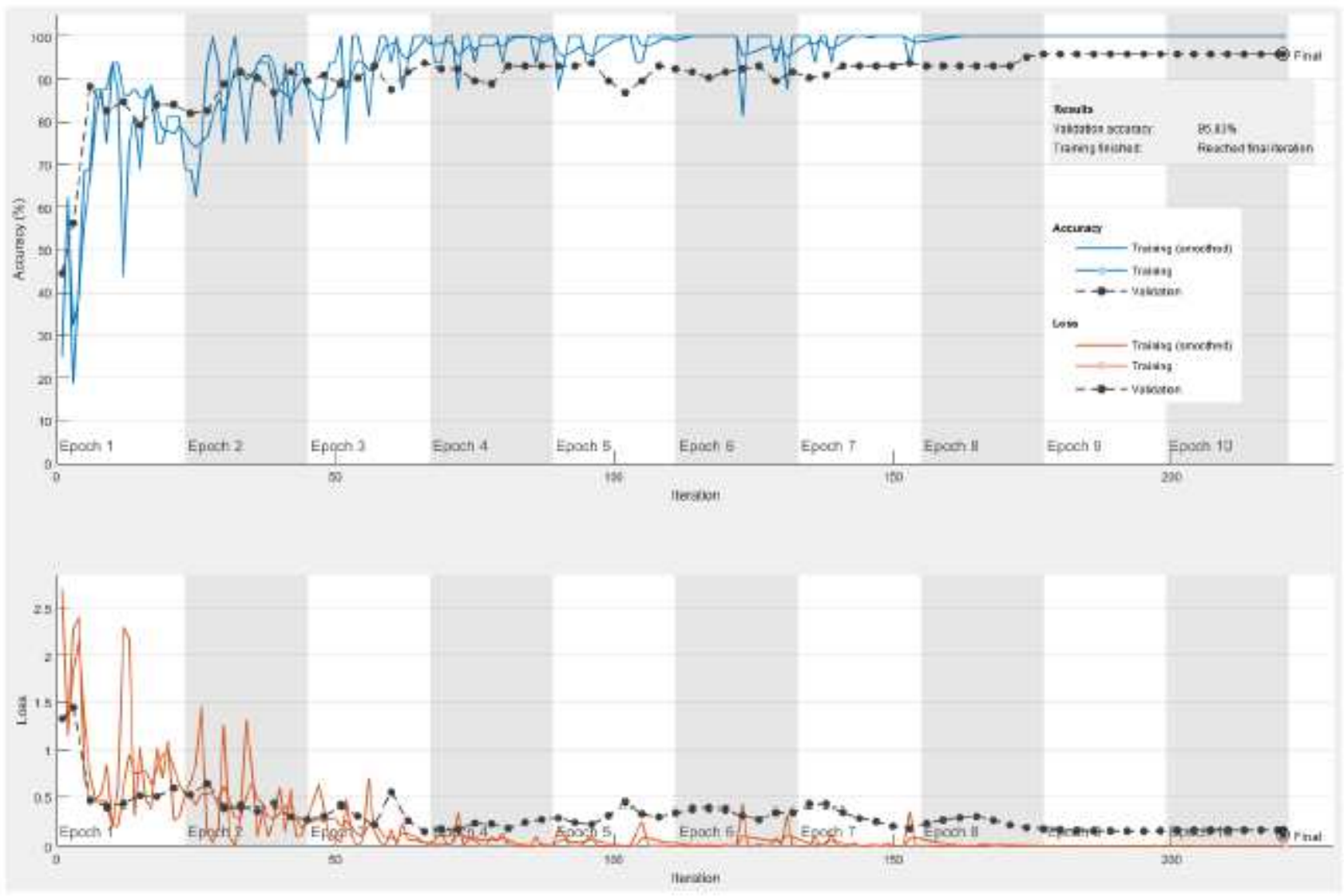

Figure 12

Training progress for ResNet18 aided method 


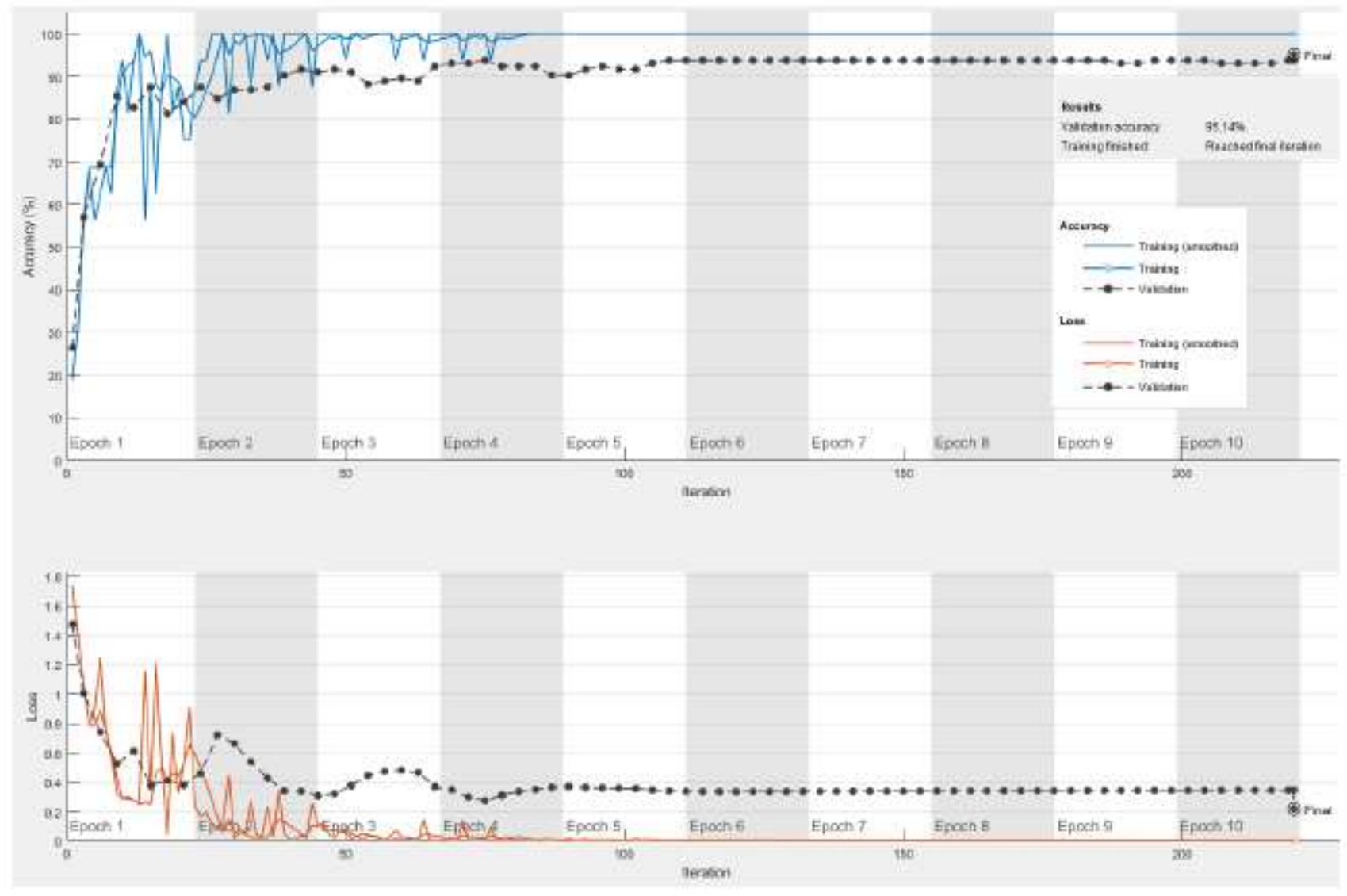

Figure 13

Training progress for ResNet50 aided method 